\title{
Accuracy and Acceptability of Oral Fluid HIV Self-Testing in a General Adult Population in Kenya
}

\author{
Ann E. Kurth ${ }^{1}$ Charles M. Cleland ${ }^{1} \cdot$ Nok Chhun ${ }^{1} \cdot$ John E. Sidle ${ }^{2} \cdot$ \\ Edwin Were $^{3,8} \cdot$ Violet Naanyu $^{4,8}$ - Wilfred Emonyi ${ }^{5,8} \cdot$ Stephen M. Macharia ${ }^{6,8}$. \\ Edwin Sang $^{8} \cdot$ Abraham M. Siika $^{7,8}$
}

Published online: 5 October 2015

(C) Springer Science+Business Media New York 2015

\begin{abstract}
We evaluated performance, accuracy, and acceptability parameters of unsupervised oral fluid (OF) HIV self-testing (HIVST) in a general population in western Kenya. In a prospective validation design, we enrolled 240 adults to perform rapid OF HIVST and compared results to staff administered OF and rapid fingerstick tests. All reactive, discrepant, and a proportion of negative results were confirmed with lab ELISA. Twenty participants were video-recorded conducting self-testing. All participants completed a staff administered survey before and after HIVST to assess attitudes towards OF HIVST acceptability. HIV prevalence was $14.6 \%$. Thirtysix of the 239 HIVSTs were invalid $(15.1 \%$; $95 \%$ CI 11.1-20.1\%), with males twice as likely to have invalid results as females. HIVST sensitivity was $89.7 \%(95 \% \mathrm{CI}$
\end{abstract}

Ann E. Kurth

akurth@nyu.edu

1 New York University College of Nursing, 433 First Avenue, 7th Floor, New York, NY 10003, USA

2 Department of General Internal Medicine, School of Medicine, Indiana University, Indianapolis, IN, USA

3 Department of Reproductive Health, School of Medicine, College of Health Sciences, Moi University, Eldoret, Kenya

4 Department of Behavioral Sciences, School of Medicine, College of Health Sciences, Moi University, Eldoret, Kenya

5 Department of Immunology, School of Medicine, College of Health Sciences, Moi University, Eldoret, Kenya

6 Moi Teaching and Referral Hospital, Eldoret, Kenya

7 Department of Medicine, School of Medicine, College of Health Sciences, Moi University, Eldoret, Kenya

8 Academic Model Providing Access to Healthcare (AMPATH), Eldoret, Kenya
73-98\%) and specificity was $98 \%$ (95\% CI 89-99\%). Although sensitivity was somewhat lower than expected, there is clear interest in, and high acceptability (94\%) of OF HIV self-testing.

Keywords HIV · Self-testing · Oral fluid · Sensitivity · Specificity

\section{Introduction}

Knowledge of HIV status is key to earlier access to HIV treatment and prevention services. As an HIV prevention strategy, HIV testing is cost effective, estimated to cost US\$249 per HIV-1 infection averted in Kenya $[1,2]$. It is also the fundamental entry point to an effective seek, test, treat and retain (STTR) paradigm, which has the potential to bend the curve of the HIV pandemic [3]. In resource limited settings such as in sub-Saharan Africa, the shortage of health care workers has been identified as a barrier in the effort to scale up HIV prevention and treatment services. Other barriers include stigma, health seeking behavior among undiagnosed persons living with HIV (PLWH), especially men, and an overemphasis on facility based approaches to HIV testing services [4]. According to population-based surveys in low- and middle-income countries (LMIC), the median percentage of people living with HIV who know their status is estimated at under $50 \%$ [5]. Given the public health implications of unknown HIV status, especially among undiagnosed PLWH, the availability of self-testing for rapid scale up of HIV testing is compelling [6]; increasing awareness of HIV status is an important step towards reducing HIV transmission and enabling antiretroviral therapy (ART) that reduces mortality as well as secondary HIV transmission. HIV testing 
guidelines recently released by the World Health Organization (July 2015) highlighted the potential contribution of HIV self-testing (HIVST) to close critical gaps in HIV testing coverage, and to support retesting efforts, worldwide [7].

Data from both blood and oral fluid HIV self-testing studies conducted in Canada [8], China [9], Kenya [10], Malawi [11], Singapore [12, 13], Spain [14, 15], South Africa [16], Uganda [17], and the U.S. [18-21] show selftesting is feasible, acceptable, and accurate. Availability of self-testing is one of several options to increase access to testing, especially in higher-risk subpopulations $[9,21]$ that may not access current forms of HIV testing, such as HIVdiscordant couples, men who have sex with men (MSM), sex workers (SWs), people who inject drugs (PWID), and high-risk youth. Currently available options include voluntary counseling and testing (VCT), provider-initiated counseling and testing (PICT), home-based counseling and testing (HBCT), self-testing home specimen collection, and mobile and outreach HIV testing and counseling. HIV selftest results do not confer diagnosis, as a reactive (positive) HIV self-test must be confirmed according to national algorithms; individuals with non-reactive (negative) HIV self-test results should be provided with information about re-testing, especially if they had a recent exposure or are at on-going risk. In the US, where approximately 1 in 5 people do not know they are infected [22], the Food and Drug Administration (FDA) approved the OraQuick InHome HIV test kit, the first HIV self-test (HIVST) kit for sale directly to consumers over-the-counter (OTC) and online [19]. However, such an approach has not yet been implemented as a standard option for non-health professionals in LMIC countries, e.g., sub-Saharan Africa, where two-thirds of all HIV infection globally exists.

Kenya has been a leader in innovative approaches to HIV prevention and care. The recent KAIS [23] national survey found that levels "of HIV testing have increased with $72 \%$ of adults aged 15-64 years in 2012 reporting ever having been tested for HIV, a significant increase from $34 \%$ in 2007." Ambitious population coverage targets for HIV serostatus knowledge have been set, yet even with largescale HBCT programs, VCT scale up, and other approaches; there still remains a coverage gap as noted by KAIS (one that is larger for men than women). This suggests HIVST has a place as part of a comprehensive testing strategy. Kenya has successfully piloted HIVST among health workers [10] and is the first African country (and one of the first countries globally) to develop policy guidelines [24] around HIVST for the general public. Our objective is to evaluate the performance and accuracy parameters of unsupervised oral fluid HIV self-testing among adult lay users in the general population of Kenya. The study aims were to determine (1) the ability of participants with unknown HIV status to correctly perform and interpret a rapid oral fluid (OF) HIV test and compare their results to staff/lab testing (i.e., sensitivity and specificity measures) and (2) participant attitudes and preferences towards $\mathrm{OF}$ self-testing (i.e., OF HIVST acceptability and feasibility).

\section{Methods}

\section{Participants}

Study participants were recruited from three sites: a health care facility that has expanded beyond HIV-only care provision and two community workplace settings in Eldoret, Kenya from November 11-29, 2013. Study flyers with mobile phone contact numbers were used to recruit at all three sites, along with provider word of mouth (clinical site) and employer information with referral to our study staff at the occupational sites. Eligibility criteria included (1) adults ( $\geq 18$ years old), (2) who do not know their HIV status because they have never tested or their last HIV test was negative, and (3) live within $1 \mathrm{~h}$ of public transit travel time to Eldoret town. Participants received 250 Kenyan shillings (Ksh, equivalent to 3.00 USD) for their time and to reimburse their transportation costs.

\section{Study Procedures}

We conducted a prospective validation study comparing unsupervised self-administered OF HIVST among $\mathrm{n}=240$ individuals to staff-administered OF and rapid fingerstick whole blood (FS) test; all reactive, discrepant, invalid, and a proportion of the negative results were validated using an enzyme-linked immunosorbent assay (ELISA). We also assessed HIVST steps to understand errors in a subset of the 240 participants, who agreed to be videotaped while performing the steps of the self-testing. All participants conducted an OF self-test, followed by staff administered OF (to separate errors due to the lower accuracy of oral specimens versus errors due to participants' lack of knowledge or experience with the self-testing kit [25] ) and FS rapid diagnostic test. A subset of 20 participants' selftesting performance was video streamed which enabled study staffers to monitor the steps, in a separate area, in real time using a standardized checklist of steps and errors noted [26]. Two study staff reviewed and compared their checklists, and resolved discrepant observations. Only participants enrolled from the health facility site were asked to be part of the videotaped self-test usability component of the study; every participant was invited at the time of informed consent until 20 were enrolled.

The study was conducted over a single session and enrollment was done with written informed consent of each 
participant. All study procedures were approved by Moi University College of Health Sciences and Moi Teaching and Referral Hospital's Institutional Research and Ethics Committee (\#1029) and NYU's University Committee on Activities Involving Human Subjects (\#13-9670). Following informed consent, study staff administered a preHIVST questionnaire to collect basic demographic information and to assess risks, HIV test history, and HIV selftesting importance, confidence, and concerns. Afterwards, participants performed the self-testing in a private space, using a pictorial instruction sheet with both English and Kiswahili language text and icons, without supervision from study staff members. The OraQuick ADVANCE Rapid HIV 1/2 Antibody Test (OraSure Technologies), a rapid diagnostic oral fluid test kit approved for use by the Kenya Ministry of Health was used. After OF self-specimen collection, participants informed study staffer they completed HIVST by interpreting their results aloud. Afterwards, study staff administered a post-HIVST questionnaire to collect information about participant experience using the self-test kit, how HIVST should be packaged or made available, possible reasons people may or may not use HIV prevention and treatment services, as well as some of the same information collected during the pre-test questionnaire. Study staff then collected an $\mathrm{OF}$ sample and a blood FS sample (The Alere Determine HIV1/2; Alere Medical Co.) and conducted parallel OF and FS rapid HIV tests. A blood specimen was drawn for ELISA confirmatory testing (Vironostika HIV Uni-Form II Ag/Ab; bioMérieux Inc.) at the time of any reactive, discrepant, or indeterminate/invalid OF/FS test results (except for one participant with an invalid OF who refused) and a percentage $(29 \%)$ of OF negative results for quality control purposes. The ELISA was selected as the reference standard as it is used in Kenya's current national guidelines (at the time of the study) as the final serostatus determination assay and we followed these guidelines for the index value/cutoff. A single laboratory staff person conducted and read the ELISA who was trained to national standards in the AMPATH lab, which ISO 15189:2007 certified. Laboratory staff person was blinded to the results of the other tests and did not have access to other clinical information. Post-test counseling was provided according to Kenya Ministry of Health guidelines, after completion of all study procedures. All participants with a reactive test result were notified of their confirmed HIV-positive status once ELISA results were available and referrals/linkage to appropriate HIV care were made.

\section{Sample Size Determination}

Assuming sensitivity is $96 \%$ and specificity is $99 \%$, and an undiagnosed HIV prevalence of $20 \%$, the total sample size of up to $\mathrm{N} \leq 240$ (minimum $\mathrm{N}=180$ ) provides adequate $(\geq 80 \%)$ power to detect key errors in HIV selftesting steps during this usability/validation pilot, including specimen collection and interpretation. For sensitivity, a null hypothesis of $S e=.80$ can be rejected with $82 \%$ power when the true value is .96 ; for specificity, a null hypothesis of $S p=.80$ can be rejected with $99 \%$ power when the true value is .99 .

For the videotaping we set out to attain a sample of 10 males and 10 females. Samples of this size have been shown to have sufficient power to detect the large majority of usability problems [27].

\section{Statistical Analysis}

Statistical analysis was conducted using Stata 13.1 (Stata Corporation, College Station, Texas, USA). Distributions of participants' characteristics and OF HIVST, FS, and ELISA laboratory results were assessed using contingency tables. With ELISA and staff FS as gold standards, the performance of the rapid OF HIVST diagnostic test was estimated using the diagt module in Stata [28]. Cohen's kappa [29] also was reported in order to convey the strength of agreement between different HIV diagnostic tests.

Data from the observation checklist for videotaped participants were entered into an excel database and data were analyzed descriptively for common user errors.

\section{Results}

We enrolled a total of 240 participants. Figure 1 outlines the status of enrollees. One study participant enrolled was HIV-positive and diagnosed prior to the study; this individual was removed from the analysis.

As seen in Table 1, the sample was approximately one third female and two-thirds males. Most participants $(90 \%)$ had tested for HIV, while $10 \%(\mathrm{n}=23)$ reported having never tested previously. A handful $(\mathrm{n}=8$ females, $3 \%$ ) said they had performed any kind of self-test before, in this case an over the counter pregnancy test. Mean age was 33, and mean education was 12 years. Two thirds of the sample were laborers. Most participants ( $88 \%)$ were in a relationship, though we saw only six out of this group who came in with a partner as part of a HIV-discordant couple.

Sexual risk-taking was common (Table 1), with $81 \%$ acknowledging sex without a condom in the last month. Of those with a main sex partner, two-thirds said their partners had been tested for HIV, with $7 \%$ of those reporting the partner's status was HIV-positive (8/9 of those were on ART). Mean number of sex partners in the last month was 


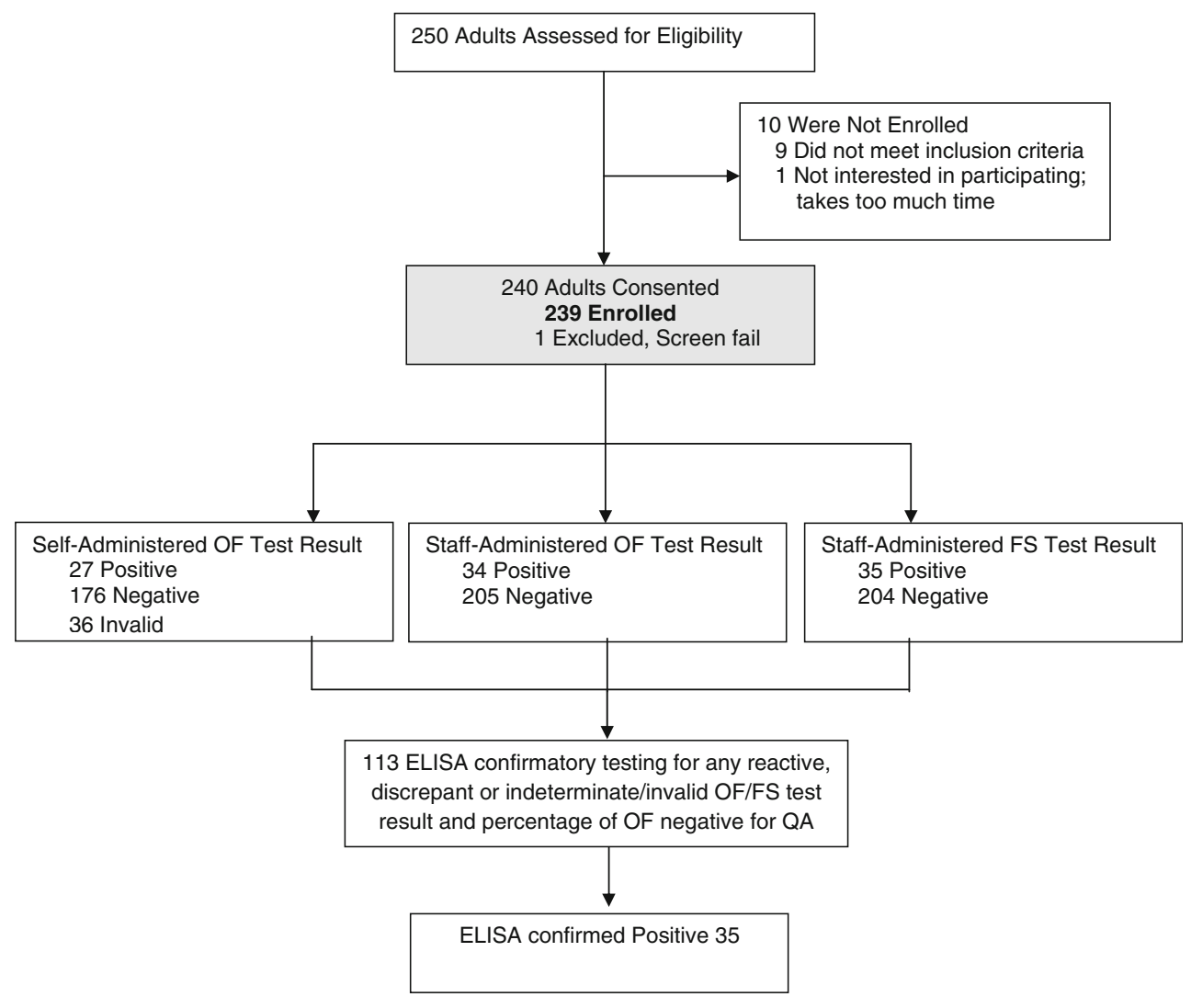

Fig. 1 Enrollment

1.35 , indicating some possible sexual concurrency, a factor thought to contribute to HIV epidemic spread. Around $6 \%$ of participants said they had traded sex for drugs, money, food, clothing, shelter, or any other goods in the last 30 days. Almost half $(45 \%)$ said they were less concerned about HIV than they used to be, though $91 \%$ acknowledged being "very worried about getting HIV; HIV stigma was still prevalent $(60 \%$ "worried about what people in the community will think if I have HIV") but not universal.

The majority had a previous history of HIV testing with a mean of 3.77 tests taken (SD 2.30), and most had tested within the last year (Table 2). Previous test sites included $\operatorname{VCT}(61 \%)$, with $31 \%$ tested at a hospital and $4 \%$ at home. Reasons for not testing included fear and perceived low risk. Concerns about testing HIV positive included stigma, abandonment and violence (all over $50 \%$ ), with confidentiality as the main/top reason for not having tested for HIV before.

Prevalence of HIV infection was based on staff FS results, since staff FS results were equivalent to the ELISA laboratory blood test (sensitivity and specificity both $100 \%$ ) and available for all participants. A total of 35 of 239 participants were positive for HIV infection, indicating prevalence of just under $15 \% \quad(14.6 \% ; 95 \% \quad \mathrm{CI}$ $10.7-19.7 \%)$.

\section{Sensitivity and Specificity}

Determination of the accuracy of OF HIV self-testing was based on comparison with the ELISA blood test and staff FS rapid HIV testing. Sensitivity was estimated as the proportion of positive ELISA results that were also positive by OF HIVST. Specificity was estimated as the proportion of negative ELISA (or staff FS) results that were also negative by OF HIVST. Among the 239 OF HIV selftesting results, $36(15.1 \%$; $95 \%$ CI 11.1-20.1\%) were invalid. More men than women had invalid tests; $7.7 \%$ $(\mathrm{n}=6)$ women and $18.6 \%(\mathrm{n}=30)$ men $(p<0.03$, OR 2.7). All individuals with an invalid test did recognize that something had gone wrong with their test and did not misinterpret it as either a negative or positive result. Given that invalids thus represented a form of missing data, and in order to document the most direct estimate of test performance, we excluded invalid test results from analysis of accuracy.

Table 3 compares positive test results by type of test; it is worth noting that the fingerstick results were the same as the lab-confirmed ELISAs in all cases where both were available. Among 29 participants with positive ELISA results, 3 false negatives were observed for OF HIVST $($ Sensitivity $=89.7 \%$; $95 \%$ CI 72.6-97.8 \%). Among 49 
Table 1 Demographic characteristics and sexual risk behaviors

\begin{tabular}{|c|c|c|c|c|}
\hline & \multicolumn{4}{|l|}{ Sites } \\
\hline & $\begin{array}{l}\text { Site } 1 \\
(\mathrm{n}=77)\end{array}$ & $\begin{array}{l}\text { Site } 2 \\
(\mathrm{n}=73)\end{array}$ & $\begin{array}{l}\text { Site } 3 \\
(\mathrm{n}=89)\end{array}$ & $\begin{array}{l}\text { Overall } \\
(\mathrm{n}=239)\end{array}$ \\
\hline \multicolumn{5}{|l|}{ Demographics } \\
\hline Age & $30.86(8.86)$ & $40.38(10.05)$ & $36.74(8.00)$ & $35.96(9.69)$ \\
\hline Gender male & $39(50.65)$ & $44(60.27)$ & $78(87.64)$ & $161(67.36)$ \\
\hline Highest education level & $12.18(3.86)$ & $11.79(2.66)$ & $12.13(2.77)$ & $12.04(3.13)$ \\
\hline \multicolumn{5}{|l|}{ Relationship status } \\
\hline Single (no current main relationship) & $14(18.18)$ & $2(2.74)$ & $13(14.61)$ & $29(12.13)$ \\
\hline Currently married (certificate/custom), one wife/husband & $38(49.35)$ & $57(78.08)$ & $34(38.20)$ & $129(53.98)$ \\
\hline Currently in a relationship but not living with partner & $18(23.38)$ & $4(5.48)$ & $1(1.12)$ & $23(9.62)$ \\
\hline \multicolumn{5}{|l|}{ Household monthly income } \\
\hline None & $16(21.05)$ & $0(0.00)$ & $1(1.12)$ & $17(7.14)$ \\
\hline \multirow[t]{2}{*}{ 0-9999 Ksh } & $30(39.47)$ & $32(43.84)$ & $3(3.37)$ & $65(27.31)$ \\
\hline & & & \multicolumn{2}{|r|}{ 31) } \\
\hline$\geq 10,000 \mathrm{Ksh}$ & $30(39.47)$ & $41(56.16)$ & $85(95.51)$ & $156(65.55)$ \\
\hline \multicolumn{5}{|l|}{ Sexual Risk Behaviors } \\
\hline \multicolumn{5}{|l|}{ Sex without condom in last 12 months } \\
\hline Yes & $58(75.33)$ & $61(84.72)$ & $73(82.02)$ & $192(80.67)$ \\
\hline \multicolumn{5}{|l|}{ One person consider as main sexual partner in past 30 days } \\
\hline Yes & $60(77.92)$ & $63(86.30)$ & $77(86.52)$ & $200(83.68)$ \\
\hline \multicolumn{5}{|l|}{ Main partner ever tested for HIV } \\
\hline Yes & $23(38.33)$ & $44(69.84)$ & $61(79.22)$ & $128(64.00)$ \\
\hline No & $8(13.33)$ & $9(14.29)$ & $10(12.99)$ & $27(13.50)$ \\
\hline I don't know & $29(48.33)$ & $10(15.87)$ & $6(7.79)$ & $45(22.50)$ \\
\hline If yes, test results were: HIV results were positive & $6(26.09)$ & $0(0.00)$ & $3(4.92)$ & $9(7.03)$ \\
\hline If positive, on ARVs? Yes & $6(100.00)$ & N/A & $2(66.67)$ & $8(88.89)$ \\
\hline Average number of sex partners, last mo $(\mathrm{N}=23)$ & $1.67(0.58)$ & $1.80(0.84)$ & $1.13(0.35)$ & $1.35(0.57)$ \\
\hline \multicolumn{5}{|c|}{$\begin{array}{l}\text { Traded sex for drugs, money, food, clothing, shelter, or any other goods in } \\
\text { the last } 30 \text { days }\end{array}$} \\
\hline Yes & 14 (18.18) & $0(0.00)$ & $0(0.00)$ & $14(5.86)$ \\
\hline
\end{tabular}

For age, highest education level, and average number of sex partners, cell contents are means with standard deviations in parentheses. For all other variables, cell contents are count of participants with percentages in parentheses

participants with negative ELISA results, 1 false positive was observed for OF HIVST (Specificity $=98.0 \% ; 95 \%$ CI 89.1-99.9\%). Among 29 participants with positive staff FS results, 3 false negatives were observed for OF HIVST (Sensitivity $=89.7 \%$; $95 \%$ CI 72.6-97.8 \%). Among 174 participants with negative staff FS results, one false positive was observed for OF HIVST (Specificity $=99.4 \%$; $95 \%$ CI 96.8-99.9\%). All participants positive by FS were confirmed by ELISA, which identified exactly the same people as having HIV infection. However, only a sample of negatives was confirmed by ELISA, hence the slight difference in specificity. When the videotaped cohort participants $(\mathrm{n}=20)$ were excluded from analysis, OF HIVST sensitivity was slightly higher (Sensitivity = $92.9 \%$; $95 \%$ CI 76.5-99.1\%), and specificity slightly lower $($ Specificity $=97.8 ; 95 \%$ CI 88.5-99.9\%).
Negative and positive predictive values for OF HIVST relative to ELISA and staff FS results were estimated as well. Among 51 participants with a negative OF HIVST result, 48 were also negative by ELISA (NPV $=94.1 \%$; $95 \%$ CI 83.8-98.8 \%). Among 27 participants with a positive OF HIVST result, 26 were also positive by ELISA (PPV $=96.3 \%$; CI 81.0-99.9\%). Among 176 participants with a negative OF HIVST result, 173 were also negative by staff FS (NPV $=98.3 \% ; \quad 95 \%$ CI 95.1-99.6\%). Among 27 participants with a positive OF HIVST result, 26 were also positive by staff FS $(\mathrm{PPV}=96.3 \%$; $95 \%$ CI 81.0-99.9\%).

Cohen's kappa was .89 (95\% CI .78-.99) for agreement between OF HIVST and ELISA and was .92 (95\% CI .84-.99) for agreement between OF HIVST and staff FS results. 
Table 2 Exposure to services, HIV test history, perceptions and concerns

\begin{tabular}{|c|c|c|c|c|}
\hline & \multicolumn{4}{|l|}{ Sites } \\
\hline & Site $1(\mathrm{n}=77)$ & Site $2(n=73)$ & Site $3(n=89)$ & Overall $(n=239)$ \\
\hline \multicolumn{5}{|l|}{ Ever tested for HIV } \\
\hline Yes & $77(100.00)$ & $63(86.30)$ & $76(85.39)$ & $216(90.38)$ \\
\hline Number of times tested for HIV & $3.55(2.02)$ & $2.48(1.38)$ & $5.04(2.53)$ & $3.77(2.30)$ \\
\hline How long ago was most recent test & $10.81(13.81)$ & $17.83(17.16)$ & $11.87(26.04)$ & $12.34(20.42)$ \\
\hline \multicolumn{5}{|l|}{ Most recent HIV test result } \\
\hline Positive & $0(0.00)$ & $1(1.59)$ & $0(0.00)$ & $1(0.46)$ \\
\hline Negative & $77(100.00)$ & $62(98.41)$ & $76(100.00)$ & $215(99.54)$ \\
\hline Didn't get result & $0(0.00)$ & $0(0.00)$ & $0(0.00)$ & $0(0.00)$ \\
\hline \multicolumn{5}{|l|}{ Location where last tested for HIV } \\
\hline VCT & $47(61.04)$ & $14(22.22)$ & $73(96.05)$ & $134(62.04)$ \\
\hline Clinical setting & $30(38.96)$ & $40(63.49)$ & $2(2.63)$ & $72(33.33)$ \\
\hline Home & $0(0.00)$ & $8(12.70)$ & $1(1.32)$ & $9(4.17)$ \\
\hline Other & $0(0.00)$ & $1(1.59)$ & $0(0.00)$ & $1(0.46)$ \\
\hline \multicolumn{5}{|l|}{ If never tested, reasons why (check all that apply) } \\
\hline I thought I had a low chance of getting HIV & $0(0.00)$ & $6(60.00)$ & $1(7.69)$ & $7(30.43)$ \\
\hline Takes too much time/inconvenient & $0(0.00)$ & $1(10.00)$ & $4(30.77)$ & $5(21.74)$ \\
\hline I am afraid of knowing that I may have HIV & $0(0.00)$ & $2(20.00)$ & $6(75.00)$ & $8(33.33)$ \\
\hline Other & $0(0.00)$ & $1(10.00)$ & $3(23.08)$ & $4(17.39)$ \\
\hline \multicolumn{5}{|l|}{ Ever used self-tests in the past } \\
\hline Yes & $7(9.09)$ & $1(1.39)$ & $0(0.00)$ & $8(3.36)$ \\
\hline No & $70(90.91)$ & $71(98.61)$ & $89(100.00)$ & $230(96.64)$ \\
\hline \multicolumn{5}{|c|}{ Concerns about testing HIV positive (check all that apply) } \\
\hline Fear of stigma & $73(94.81)$ & $42(57.53)$ & $41(46.07)$ & $156(65.27)$ \\
\hline Fear of abandonment & $72(93.51)$ & $32(43.84)$ & $44(49.44)$ & $148(61.93)$ \\
\hline Fear of family violence & $37(48.05)$ & $53(72.60)$ & $27(30.34)$ & $117(48.95)$ \\
\hline Confidentiality concerns & $70(90.91)$ & $54(73.97)$ & $36(40.45)$ & $160(66.95)$ \\
\hline Access to treatment if you are HIV positive & $11(14.29)$ & $65(89.04)$ & $9(10.11)$ & $85(35.57)$ \\
\hline Other & $2(2.60)$ & $9(12.33)$ & $28(31.46)$ & 39 (16.32) \\
\hline
\end{tabular}

For number of times tested and time since the most recent test, cell contents are means with standard deviations in parentheses. For all other variables, cell contents are count of participants with percentages in parentheses

\section{Test Performance Video Data}

Twenty individuals were videotaped during their HIVST. Video observation demonstrated that all participants reviewed the instruction sheet prior to performing the selftest. Problems noted covered a range of issues, some minor (e.g., twisting rather than popping the cap, $\mathrm{n}=14$ ) and others less so (e.g., placing swab in the buffer solution before sample collection, $n=3$ ). These errors are similar to those that have been noted in a 3-country videotaped HIVST study conducted by PATH [26]. In addition, although not captured during the videotaping session, or from the post-HIVST questionnaire, research assistant field notes anecdotally describe one participant drinking the buffer solution. Outcomes from the test performance video data are summarized in Table 4. The same individual could have made multiple errors.

\section{Invalids}

By design, samples positive by OF HIVST were oversampled for ELISA confirmatory testing, so it is not feasible to use ELISA results to compare HIV prevalence among participants with and without invalid OF HIVST results. When staff finger-stick results (available for all participants) are used as a gold standard for HIV infection, those with invalid self-testing results had slightly increased odds of infection, but the difference was not statistically significant $(\mathrm{OR}=1.20 ; p=0.7977$ by Fisher's Exact Test). 
Table 3 Results by type of test

\begin{tabular}{|c|c|c|c|}
\hline & \multicolumn{2}{|c|}{ Staff finger stick result } & \multirow[t]{2}{*}{ Total } \\
\hline & Negative & Positive & \\
\hline \multicolumn{4}{|c|}{ Participant oral fluid self-test result } \\
\hline Invalid $^{\mathrm{a}}$ & 30 & 6 & 36 \\
\hline Negative & 173 & 3 & 176 \\
\hline Positive & 1 & 26 & 27 \\
\hline Total & 204 & 35 & 239 \\
\hline \multicolumn{4}{|c|}{ Staff oral fluid result } \\
\hline Invalid $^{\mathrm{a}}$ & 0 & 0 & 0 \\
\hline Negative & 204 & 1 & 205 \\
\hline Positive & 0 & 34 & 34 \\
\hline Total & 204 & 35 & 239 \\
\hline \multicolumn{4}{|c|}{$\begin{array}{l}\text { Excluding } \quad \text { invalid } \\
(\text { Sensitivity }=89.7 \%)\end{array}$} \\
\hline \multicolumn{4}{|c|}{ Excluding invalid results, one false positive $($ Specificity $=98.0 \%$ ) } \\
\hline
\end{tabular}

In our study sample, participants recruited from Site 3 had increased odds of an invalid test result $(\mathrm{OR}=6.76$; $p<0.0001$ by Fisher's Exact Test), and being male also increased the odds of an invalid test result $(\mathrm{OR}=2.74$; $p=0.0329$ by Fisher's Exact Test). Age ( $>25$ years) and education ( $<10$ years) were not significantly associated with the odds of an invalid OF HIVST result (OR = 2.13; $p=0.3129$ by Fisher's Exact Test, and $\mathrm{OR}=1.92$; $p=0.1179$, by Fisher's Exact Test, respectively). Participants who had never tested before had increased odds of an invalid result, but this finding was only marginally significant $(\mathrm{OR}=2.81 ; p=0.0579$ by Fisher's Exact Test).

\section{HIVST Acceptability}

As seen in Table 5, results drawn from the post-HIVST behavioral questionnaire closed-ended/quantified questions, the importance of having access to a HIVST increased before vs. after the person conducted their own self-test,
Table 4 List of HIVST user errors

\begin{tabular}{|c|c|}
\hline Video observation user errors & $\mathbf{N}$ \\
\hline \multicolumn{2}{|l|}{ Test preparation } \\
\hline Difficulty opening packet & 2 \\
\hline Difficulty opening bottle & 14 \\
\hline Difficulty putting bottle in stand (holder)/Did not put bottle in stand & 5 \\
\hline Placed stand in wrong position & 2 \\
\hline Placed swab in stand & 2 \\
\hline Placed swab on table & 6 \\
\hline \multicolumn{2}{|l|}{ Sample collection } \\
\hline Did not swab between teeth and gums & 1 \\
\hline Touched bottom of swab with fingers & 2 \\
\hline Tongue touched swab & 1 \\
\hline Holds swab in mouth & 1 \\
\hline Used finger to swab with desiccant & 1 \\
\hline Collected sample with finger instead of using swab & 2 \\
\hline Placed swab in buffer solution before collecting sample & 2 \\
\hline Placed swab in buffer solution without collecting sample & 1 \\
\hline Placed finger in buffer solution before collecting sample with finger & 1 \\
\hline \multicolumn{2}{|l|}{ General other errors } \\
\hline Added desiccant to solution & 2 \\
\hline Used swab as stirrer & 1 \\
\hline Spilled solution & 2 \\
\hline Poured solution into stand & 1 \\
\hline Spits into bottle & 1 \\
\hline Illiteracy & 2 \\
\hline Did not keep swab in bottle for the entire time & 3 \\
\hline \multicolumn{2}{|l|}{ Timing } \\
\hline Waited $<20 \mathrm{~min}$ to read results & 13 \\
\hline
\end{tabular}


going from a mean of 8.1-8.6 (ascending scale 0-10). Confidence in the ability to perform and interpret the selftest appeared to increase with exposure to actually doing the test. Confidence in doing the self-test correctly rose from 6.0 to 7.9 and ability to read results correctly from 6.1 to 8.1. Only 11 participants (4.6\% overall) thought that doing the HIVST was 'very difficult or difficult' while $94 \%$ 'strongly agreed or agreed' that HIVST was acceptable.

Affordability was a main theme in the behavioral survey data, mentioned by $n=192 / 239$. The mean price people were willing to pay for a HIVST was $111 \mathrm{Ksh}$, around US\$1.25 (range 0-1000 Ksh). Female mean price was 78 $\mathrm{Ksh}$ and male mean was $158 \mathrm{Ksh}$, though median price was $50 \mathrm{Ksh}$ for both. The mean price among those under age 25 was $56 \mathrm{Ksh}$ and for those 25 and older was $120 \mathrm{Ksh}$. (Of note, at the time of the study, the cost of the Oraquick selftest kit was US\$7.50 per kit or $\sim 600$ Kenyan shillings at an exchange rate of 80 . However, the Oraquick test is not yet publicly available for use in Kenya).

\section{Discussion}

This HIV self-test validation study conducted among a general population sample of unsupervised lay users in western Kenya found a sensitivity of 89.7-92.9\% and specificity of 97.8-98.0\%. Agreement between OF HIVST and both ELISA and staff FS results was very good. Of note, around one in seven users had invalid results. The study population had an overall HIV prevalence of just under $15 \%$. By comparison, the KAIS 2012 national household survey found HIV prevalence in the North Rift Province where the study took place (Eldoret is in Uasin
Gishu County) was $3.1 \%$. The higher prevalence seen in our sample as compared with that found in the province during the KAIS 2012 survey suggests somewhat higherrisk individuals self-selected to participate in the study, which may be a selection bias but that at least indicates an interest in HIVST among this epidemiologically and clinically important subgroup of the general adult population.

Of note, $7.7 \%(n=6)$ women and $18.6 \%(n=30)$ of the men had invalid HIVSTs. This may suggest less familiarity with health tests among men. Potentially, factors such as literacy and previous exposure to health tests may influence HIVST performance, perhaps suggesting the need for educational campaigns and improved instructions for the self-test kits, particularly for men who had an invalid test rate more than double that of women.

Study limitations include the small sample size and potential volunteer bias. Power to detect differences in sensitivity or specificity based on participant characteristics is low. The sample size for cases with infection is 29 , and for cases without infection is 49 . Also, because the number of diagnostic errors among those with a valid OF HIVST result is very small (one false positive and three false negatives), it is nearly impossible to identify statistically significant predictors of diagnostic error/accuracy.

Supervised oral self-testing in Malawi found an overall sensitivity of $97.9 \%(95 \%$ CI $87.9 \%-100.0 \%)$ and specificity of $100 \%$ (95\% CI $97.8 \%-100.0 \%$ ) [11]. Although sensitivity in our study was lower than expected in comparison, in the overall sample it was nearly $90 \%$ for unsupervised HIVST, similar to the $90 \%$ reported for an unsupervised HIVST study conducted in Uganda where each participant first received a 10-minute HIVST demonstration [17]. When the videotaped subjects were

Table 5 Pre and post HIVST importance, confidence, and acceptability

\begin{tabular}{|c|c|c|c|c|c|c|c|c|}
\hline & \multicolumn{8}{|l|}{ Sites } \\
\hline & \multicolumn{2}{|c|}{ Site $1(n=77)$} & \multicolumn{2}{|c|}{ Site $2(n=73)$} & \multicolumn{2}{|c|}{ Site $3(n=89)$} & \multicolumn{2}{|c|}{ Overall $(\mathrm{n}=239)$} \\
\hline & Pre & Post & Pre & Post & Pre & Post & Pre & Post \\
\hline $\begin{array}{l}\text { Importance of having access to an HIV self-test } \\
\text { kit }\end{array}$ & $\begin{array}{l}8.23 \\
(1.05)\end{array}$ & $\begin{array}{l}8.90 \\
(0.80)\end{array}$ & $\begin{array}{l}9.51 \\
(1.20)\end{array}$ & $\begin{array}{l}9.77 \\
(0.49)\end{array}$ & $\begin{array}{l}6.82 \\
(2.19)\end{array}$ & $\begin{array}{l}7.37 \\
(2.48)\end{array}$ & $\begin{array}{l}8.10 \\
(1.95)\end{array}$ & $\begin{array}{l}8.59 \\
(1.89)\end{array}$ \\
\hline $\begin{array}{l}\text { Confident that will use/used the self-test } \\
\text { correctly }\end{array}$ & $\begin{array}{l}3.10 \\
(2.27)\end{array}$ & $\begin{array}{l}7.99 \\
(1.36)\end{array}$ & $\begin{array}{l}8.37 \\
(0.99)\end{array}$ & $\begin{array}{l}8.48 \\
(0.97)\end{array}$ & $\begin{array}{l}6.82 \\
(2.04)\end{array}$ & $\begin{array}{l}7.34 \\
(2.65)\end{array}$ & $\begin{array}{l}6.09 \\
(2.86)\end{array}$ & $\begin{array}{l}7.90 \\
(1.92)\end{array}$ \\
\hline $\begin{array}{l}\text { Confident that will be able/read results } \\
\text { correctly }\end{array}$ & $\begin{array}{l}2.97 \\
(2.24)\end{array}$ & $\begin{array}{l}8.26 \\
(1.61)\end{array}$ & $\begin{array}{l}8.18 \\
(1.02)\end{array}$ & $\begin{array}{l}8.59 \\
(0.80)\end{array}$ & $\begin{array}{l}7.16 \\
(2.16)\end{array}$ & $\begin{array}{l}7.64 \\
(2.69)\end{array}$ & $\begin{array}{l}6.13 \\
(2.92)\end{array}$ & $\begin{array}{l}8.14 \\
(1.96)\end{array}$ \\
\hline Think/feel using this test will be/was ${ }^{\mathrm{a}}$ & 2.60 & 0.00 & 5.48 & 0.00 & 5.62 & 12.36 & 4.60 & 4.60 \\
\hline $\begin{array}{l}\text { Think/feel that HIV self-testing will be/is } \\
\text { acceptable }^{\mathrm{b}}\end{array}$ & 100.00 & 100.00 & 100.00 & 100.00 & 87.64 & 84.27 & 95.40 & 94.14 \\
\hline
\end{tabular}

a Responses have been dichotomized as percentage who said using HIVST was 'very difficult or difficult' (vs. 'not difficult, easy, very easy')

${ }^{\mathrm{b}}$ Responses have been dichotomized as percentage who 'strongly agreed or agreed' HIVST was acceptable (vs. strongly disagree, disagreed, or neither agreed or disagreed) 
excluded, sensitivity (92.9\%) was similar to that reported to the Food and Drug Administration (phase 3, $92.9 \%$ ) when they approved self-testing for general population use in the US. In our study, positive predictive value at this prevalence was reasonable at $96 \%$, though negative predictive value was lower at $94.1 \%$. Acceptability of HIVST was high (94\%) and similar to what was found in studies conducted in Malawi [11], Singapore [12], and the US [18]. In conclusion, there is clear interest in, and good acceptability of, HIV self-testing among the general population in western Kenya that may help expand knowledge of serostatus. Any rollout of HIVST should be accompanied by education around appropriate use to reduce invalid results and to encourage confirmatory testing and linkage to HIV care where appropriate.

Acknowledgments We thank the following deeply for their contributions and support: David Resto at New York University, Freya Spielberg at George Washington University; Roger Peck and Jeanette Lim at PATH; our research assistants, Titus Kipkorir Komen, Carolyne Chepkosgei Kurgat, Eunice Jepkorir Sing'oei, Anne Chepleting Tarus, Irene Khanali Wafula, and Eric Wangwelo; Judah Kimuge at AMPATH/Moi University School of Medicine for performing the laboratory assays; and the study participants. Research discussed in this publication has been funded by the International Initiative for Impact Evaluation, Inc. (3ie). The views expressed in this article are not necessarily those of 3ie or its members.

\section{References}

1. Menzies N, Abang B, Wanyenze R, et al. The costs and effectiveness of four HIV counseling and testing strategies in Uganda. Aids. 2009;23(3):395-401.

2. Sweat M, Gregorich S, Sangiwa G, et al. Cost-effectiveness of voluntary HIV-1 counselling and testing in reducing sexual transmission of HIV-1 in Kenya and Tanzania. Lancet. 2000;356(9224):113-21.

3. Granich RM, Gilks CF, Dye C, De Cock KM, Williams BG. Universal voluntary HIV testing with immediate antiretroviral therapy as a strategy for elimination of HIV transmission: a mathematical model. Lancet. 2009;373(9657):48-57.

4. WHO. Towards universal access: scaling up priorities HIV/AIDS interventions in the health sector: progress report. 2010. Available at http://whqlibdoc.who.int/publications/2010/9789241500 395_eng.pdf. Accessed 12 Nov 2014.

5. UNAIDS. The Gap Report. 2014. Available at http://www. unaids.org/sites/default/files/media_asset/UNAIDS_Gap_report_ en.pdf. Accessed 5 Jan 2015.

6. Napierala Mavedzenge S, Baggaley R, Corbett EL. A review of self-testing for HIV: research and policy priorities in a new era of HIV prevention. Clinical Infect Dis. 2013;57(1):126-38.

7. World Health Organization (WHO). July 2015. Consolidated Guidelines on HIV Testing Services, 5Cs: Consent, Confidentiality, Counselling, Correct Results and Connection. Available at http://apps.who.int/iris/bitstream/10665/179870/1/9789241508926_ eng.pdf?ua=1. Accessed 23 Sep 2015.

8. Pant Pai N, Bhargava M, Joseph L, et al. Will an unsupervised self-testing strategy be feasible to operationalize in canada? results from a pilot study in students of a large Canadian
University. AIDS Res Treat. 2014;2014:747619. doi:10.1155/ 2014/747619.

9. Marley G, Kang D, Wilson EC, et al. Introducing rapid oral-fluid HIV testing among high risk populations in Shandong, China: feasibility and challenges. BMC Public Health. 2014;14:422.

10. Kalibala S, Tun W, Cherutich P, Nganga A, Oweya E, Oluoch P. Factors associated with acceptability of HIV self-testing among health care workers in Kenya. AIDS Behav. 2014;18(Suppl 4):S405-14.

11. Choko AT, Desmond N, Webb EL, et al. The uptake and accuracy of oral kits for HIV self-testing in high HIV prevalence setting: a cross-sectional feasibility study in Blantyre, Malawi. PLoS Med. 2011;8(10):e1001102.

12. Ng OT, Chow AL, Lee VJ, et al. Accuracy and user-acceptability of HIV self-testing using an oral fluid-based HIV rapid test. PLoS ONE. 2012;7(9):e45168.

13. Lee VJ, Tan SC, Earnest A, Seong PS, Tan HH, Leo YS. User acceptability and feasibility of self-testing with HIV rapid tests. J Acquir Immune Defic Syndr. 2007;45(4):449-53.

14. Belza MJ, Rosales-Statkus ME, Hoyos J, et al. Supervised bloodbased self-sample collection and rapid test performance: a valuable alternative to the use of saliva by HIV testing programmes with no medical or nursing staff. Sex Transm Infect. 2012;88(3): 218-21.

15. de la Fuente L, Rosales-Statkus ME, Hoyos J, et al. Are participants in a street-based HIV testing program able to perform their own rapid test and interpret the results? PLoS ONE. 2012;7(10): e46555.

16. Pant Pai N, Behlim T, Abrahams L, et al. Will an unsupervised self-testing strategy for hiv work in health care workers of south africa? a cross sectional pilot feasibility study. PloS ONE. 2013;8(11):e79772.

17. Asiimwe S, Oloya J, Song X, Whalen CC. Accuracy of un-supervised versus provider-supervised self-administered hiv testing in Uganda: a randomized implementation trial. AIDS Behav. 2014;18(12):2477-84.

18. Gaydos CA, Hsieh YH, Harvey L, et al. Will patients "opt in" to perform their own rapid HIV test in the emergency department? Ann Emerg Med. 2011;58(1 Suppl 1):S74-8.

19. FDA OraQuick in-home HIV test kit. Available at http://www. fda.gov/BiologicsBloodVaccines/BloodBloodProducts/Approved Products/PremarketApprovalsPMAs/ucm310436.htm. Summary of safety and effectiveness. 2012. Available at http://www.fda. gov/downloads/BiologicsBloodVaccines/BloodBloodProducts/ ApprovedProducts/PremarketApprovalsPMAs/UCM312534.pdf. Accessed 12 Nov 2014.

20. Nour S, Hsieh YH, Rothman RE, et al. Patients can accurately perform their own rapid hiv point-of-care test in the emergency department. Point of care. 2012;11(4):176-9.

21. Carballo-Dieguez A, Frasca T, Dolezal C, Balan I. Will gay and bisexually active men at high risk of infection use over-thecounter rapid HIV tests to screen sexual partners? J Sex Res. 2012;49(4):379-87.

22. CDC new hope for stopping HIV. CDC vital signs. 2011. Available at http://www.cdc.gov/VitalSigns/pdf/2011-12-vitalsigns. pdf. Accessed 12 Nov 2014.

23. NASCOP Kenya AIDS indicator survey 2012. Nairobi, Kenya. 2014.

24. NASCOP Guidelines for HIV Testing and Counseling in Kenya. Nairobi: Kenya. 2009.

25. Pant Pai N, Balram B, Shivkumar S, et al. Head-to-head comparison of accuracy of a rapid point-of-care HIV test with oral versus whole-blood specimens: a systematic review and metaanalysis. Lancet Infect Dis. 2012;12(5):373-80.

26. Peck RB, Lim JM, van Rooyen H, et al. What should the ideal HIV self-test look like? a usability study of test prototypes in 
unsupervised HIV self-testing in Kenya, Malawi, and South Africa. AIDS Behav. 2014;18(4):422-32.

27. Faulkner L. Beyond the five-user assumption: benefits of increased sample sizes in usability testing. Behav Res Methods Instrum Comput. 2003;35(3):379-83.

28. Seed P. DIAGT: Stata module to report summary statistics for diagnostic tests compared to true disease status. Statistical
Software Components, S423401, Boston College Department of Economics, revised 19 Feb 2010. 2001.

29. Cohen J. A coefficient of agreement for nominal scales. Educ Psychol Meas. 1960;20(1):37-46. 